\title{
Elliptical dichroism: operating principle of planar chiral metamaterials
}

\author{
Sergei V. Zhukovsky, ${ }^{1, *}$ Andrey V. Novitsky, ${ }^{2}$ and Vladimir M. Galynsky ${ }^{2}$ \\ ${ }^{1}$ Theoretical Nano-Photonics, Institute of High-Frequency and Communication Technology, Faculty of Electrical, \\ Information and Media Engineering, University of Wuppertal, Rainer-Gruenter-Strasse 21, \\ D-42119 Wuppertal, Germany \\ ${ }^{2}$ Department of Theoretical Physics, Belarusian State University, Nezavisimosti Avenue 4, 220030 Minsk, Belarus \\ *Corresponding author: sergei@uni-wuppertal.de
}

Received March 24, 2009; revised May 3, 2009; accepted May 15, 2009; posted June 1, 2009 (Doc. ID 109206); published June 24, 2009

We employ a homogenization technique based on the Lorentz electronic theory to show that planar chiral structures (PCSs) can be described by an effective dielectric tensor similar to that of biaxial elliptically dichroic crystals. Such a crystal is shown to behave like a PCS insofar as it exhibits its characteristic optical properties, namely, corotating elliptical polarization eigenstates and asymmetric, direction-dependent transmission for left- or right-handed incident wave polarization. (c) 2009 Optical Society of America OCIS codes: $050.2065,160.1245,160.1585,160.1190,350.3618,050.6624$.

Metamaterials show promise for a wide range of unusual physical phenomena rare or absent in nature $[1,2]$, including giant optical activity [2-6]. Following a pioneering work by the group of Zheludev and coworkers in 2006 [7], planar chiral structures (PCSs) were recently introduced as a distinct class of metamaterials. They consist of planar elements [7-9] that have a sense of twist and cannot be superimposed on their in-plane mirror image. PCSs are asymmetric in electromagnetic wave propagation for right-handed $(\mathrm{RH})$ versus left-handed $(\mathrm{LH})$ circularly polarized incident waves. Unlike 3D or bilayer chiral metamaterials $[4,6,10]$, which resemble bi-isotropic or gyrotropic media, PCSs change their properties if the wave propagation direction is reversed $[7,11]$. They also differ from Faraday media (also known to have related asymmetry) because PCSs have corotating elliptical polarization eigenstates $[7,12]$, while in bi-isotropic or Faraday media the eigenstates always come in pairs of $\mathrm{RH}$ and $\mathrm{LH}$ polarizations.

In this Letter, we interpret optical properties of PCSs in terms of elliptical dichroism. The properties of chiral metamaterials are usually investigated on the level of resonant electromagnetic response in an individual element [8-13]. This heavily depends on the element shape, a wide variety of which were reported lately [7-9,12,13]. Although a recent Letter [13] offers a generalized explanation based on polarization-sensitive excitation of electric and magnetic dipoles, such a treatment should still be performed separately for different PCS designs. Thus, there is a need for a macroscopic description that would make planar chirality available for studies on an abstract crystallographic level, similar to other optical phenomena like birefringence or optical activity.

The idea of regarding elliptical dichroism as a mechanism behind the optical manifestation of planar chirality is suggested by earlier experimental accounts of circular dichroism in PCSs $[7,13]$. We confirm its existence by using the Lorentz-theory homogenization scheme in chiral split-ring (CSR) structures [13-15]. Moreover, we show that a bulk elliptically dichroic medium exhibits all the characteristic properties of PCS, namely, (i) corotating elliptical polarization eigenstates, (ii) asymmetric transmission for $\mathrm{RH}$ versus $\mathrm{LH}$ circular polarization, and (iii) enantiomeric asymmetry and change of properties for different wave propagation direction.

We begin by considering a CSR unit cell [Fig. 1(b)]. For planar (as opposed to 3D) elements, the electric response is dominant because the magnetic moment is parallel to the wave propagation direction [7]. Hence, one can arrive at homogenized material properties by considering the response within the framework of the electronic Lorentz theory by using $\hat{\varepsilon}_{\text {eff }} \mathbf{E}$ $=\mathbf{E}-(4 \pi / i \omega) e\langle N \mathbf{v}\rangle . N$ and $\mathbf{v}$ are the electron concentration and velocity [16], which are positiondependent in a structured material. To derive the effective dielectric tensor $\hat{\varepsilon}_{\text {eff }}$, one needs to average over the unit cell. If $d \ll R$, the electrons in the ring can be mobile only in the azimuthal direction; so $\mathbf{v}_{m}=R \dot{\varphi} \mathbf{e}_{\varphi}$

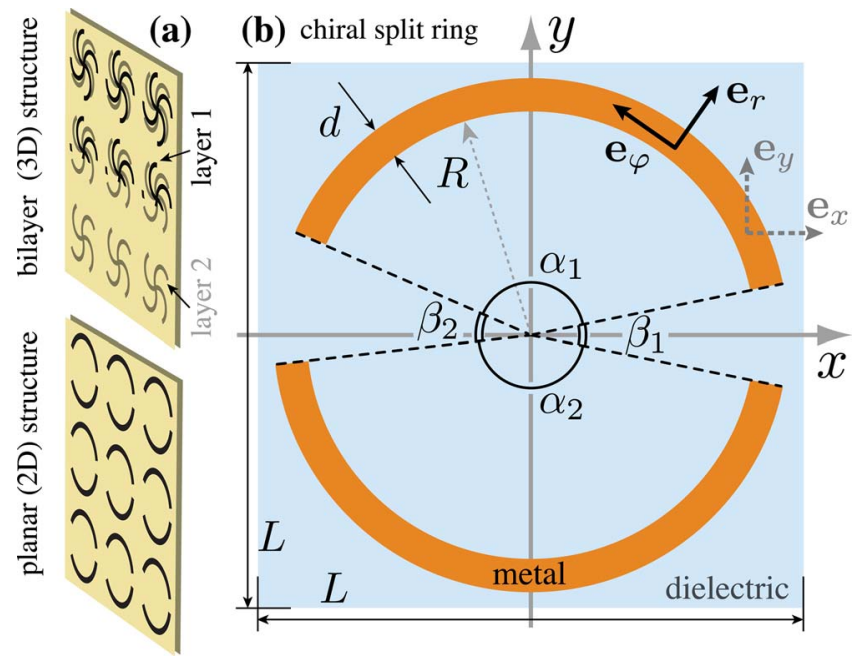

Fig. 1. (Color online) (a) Bilayer (3D) versus monolayer (planar) chiral metamaterial. (b) Unit cell of a CSR structure [13]. 
and $\ddot{\varphi}+\gamma \dot{\varphi}+\omega_{0}^{2} \varphi=\left(e / R m_{e}\right) \mathbf{e}_{\varphi} \cdot \mathbf{E}_{m}$. The electrons in the dielectric substrate are bound, and their velocity $\mathbf{v}_{d}$ in response to the electric field is position independent. The averaging is then performed as

$$
\langle N \mathbf{v}\rangle=L^{-2}\left(\int_{\text {metal }} N_{m} \mathbf{v}_{m}(\varphi) \mathrm{d}^{2} \mathbf{r}+\int_{\text {diel }} N_{d} \mathbf{v}_{d} \mathrm{~d}^{2} \mathbf{r}\right) .
$$

For the structure shown in Fig. 1, integration in Eq. (1) results in a complex symmetric tensor $\hat{\varepsilon}_{\text {eff: }}$ :

$$
\hat{\varepsilon}_{\text {eff }}(\omega)=\left[\begin{array}{ccc}
\varepsilon_{x} & \varepsilon_{x y} & 0 \\
\varepsilon_{x y} & \varepsilon_{y} & 0 \\
0 & 0 & \varepsilon_{z}
\end{array}\right] .
$$

Its components depend on the dielectric constants of metal $\varepsilon_{m}(\omega)$ and dielectric $\varepsilon_{d}(\omega)$, as well as on the geometrical parameters, e.g., the angles $\alpha_{1,2}$ and $\beta_{1,2}$ (Fig. 1). Figure 2 shows the dependencies $\varepsilon_{x, y, x y}(\omega)$. If the split ring is symmetric $\left(\beta_{2}=0, \alpha_{1}=\alpha_{2}\right.$, or $\left.\beta_{1}=\beta_{2}\right)$, then $\varepsilon_{x y}=0$ and tensor (2) supports two linearly polarized eigenwaves that are different in their attenuation (linear dichroism). Such CSRs display no planar chirality $[14,15]$. Otherwise, $\varepsilon_{x y} \neq 0$ and the dichroism is elliptical. We further rewrite the tensor $\hat{\varepsilon}_{\text {eff }}$ in axial representation [17] as

$$
\hat{\varepsilon}=\varepsilon_{o}+\left(\varepsilon_{e}-\varepsilon_{o}\right) \mathbf{c} \otimes \mathbf{c}, \quad \mathbf{c}=\mathbf{c}^{\prime}+\mathrm{i} \kappa \mathbf{c}^{\prime \prime},
$$

where the vectors $\mathbf{c}^{\prime}$ and $\mathbf{c}^{\prime \prime}$ determine the optical axes, and $\mathbf{c} \otimes \mathbf{c}$ denotes the outer dyadic product (c $\otimes \mathbf{c})_{i j} \equiv c_{i} c_{j}$.

At any fixed frequency, the medium's polarization eigenstates can be recovered from its normal refraction tensor $\mathcal{N}_{H}$ [18]. For nonmagnetic media and normal incidence

$$
\mathcal{N}_{H}^{2}=-\mathbf{q}^{\times} \hat{\varepsilon} \mathbf{q}^{\times}+(\mathbf{q} \hat{\varepsilon} \mathbf{q})^{-1} \mathbf{q}^{\times} \hat{\varepsilon} \mathbf{q} \otimes \mathbf{q} \hat{\varepsilon} \mathbf{q}^{\times},
$$

where $\mathbf{q}$ is a unit vector normal to the plane and $\mathbf{q}^{\times}$ is defined as $\left(\mathbf{q}^{\times}\right) \mathbf{u} \equiv \mathbf{q} \times \mathbf{u}$. The eigenvectors $\mathbf{H}_{1,2}$ of $\mathcal{N}_{H}^{2}$ represent the polarization states of the field that are preserved as the wave propagates along $\mathbf{q}$. If the polarization is circular or elliptical, the handedness of $\mathbf{H}_{j}$ is determined by the sign of the product $\mathbf{q} \cdot\left[\operatorname{Re} \mathbf{H}_{j} \times \operatorname{Im} \mathbf{H}_{j}\right]$ [17]. The condition for corotation of polarization eigenstates then becomes

$$
\eta=\left(\mathbf{q} \cdot\left[\operatorname{Re} \mathbf{H}_{1} \times \operatorname{Im} \mathbf{H}_{1}\right]\right)\left(\mathbf{q} \cdot\left[\operatorname{Re} \mathbf{H}_{2} \times \operatorname{Im} \mathbf{H}_{2}\right]\right)>0 .
$$
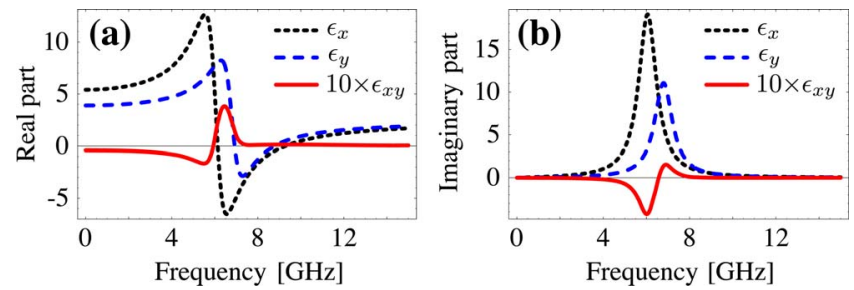

Fig. 2. (Color online) Frequency dependencies for (a) real and (b) imaginary parts of $\hat{\varepsilon}_{\text {eff }}$ in Eq. (2) for $L=15 \mathrm{~mm}, R$ $=6 \mathrm{~mm}, d=0.8 \mathrm{~mm}, \alpha_{1}=140^{\circ}, \alpha_{2}=160^{\circ}, \beta_{1}=40^{\circ}, \beta_{2}=20^{\circ}$.
For anisotropic media without absorption, $\eta=0$ (the eigenstates have linear polarization). For biisotropic chiral or Faraday media, it can be shown that $\eta<0$, so circular or elliptical polarization eigenstates are counterrotating. However, for $\hat{\varepsilon}$ of Eq. (3), where $\quad \mathbf{c}^{\prime} \equiv \mathbf{e}_{x} \cos \varphi+\mathbf{e}_{y} \sin \varphi \quad$ and $\quad \mathbf{c}^{\prime \prime} \equiv \mathbf{e}_{x} \cos \psi$ $+\mathbf{e}_{y} \sin \psi$, Eqs. (4) and (5) result in

$$
\eta=\frac{4 \kappa^{2} \sin ^{2}(\phi-\psi)}{\left(1+\kappa^{2}\right)^{2}-\left(\cos 2 \phi+\kappa^{2} \cos 2 \psi\right)^{2}} \geqslant 0 .
$$

Figure 3 shows the dependence of $\eta$ on the orientation of the axes $\mathbf{c}^{\prime}$ and $\mathbf{c}^{\prime \prime}$. One can see that polarization eigenstates are indeed corotating. They reverse their handedness at the line $\phi=\psi$. Including gyrotropy in $\hat{\varepsilon}$ would result in a negative addition to $\eta$, thus counteracting planar chirality [12].

Next, we consider the transmission spectra of an elliptically dichroic layer, which can be obtained by constructing the wave evolution operator in a generalized transfer matrix formalism [18]. Figure 4 shows the spectra for the LH and the RH circularly polarized incident wave. The transmission of $\mathrm{LH}$ versus $\mathrm{RH}$ polarization is clearly asymmetric. For higher frequencies, the spectra become flat. This is caused by the absence of material dispersion (the Lorentzian resonance is assumed to be broad), so that spectrally uniform elliptical dichroism results in spectrally uniform planar chiral behavior. Real PCSs are dispersive and thus display planar chirality only in a narrow spectral range close to resonance (see Fig. 2), in accordance with earlier findings $[7,13]$.
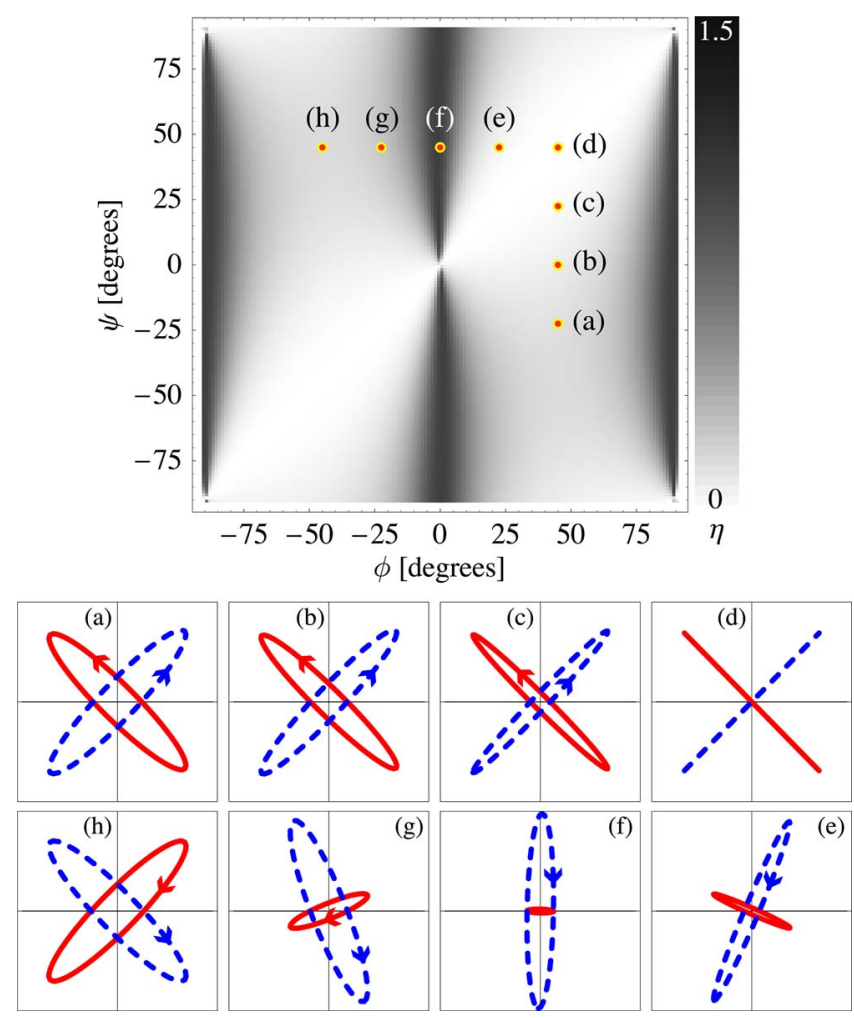

Fig. 3. (Color online) The dependence $\eta(\phi, \psi)$ as in Eq. (6) for $\kappa=0.2$ with polarization eigenstates given by Eq. (4) at points (a)-(h). 


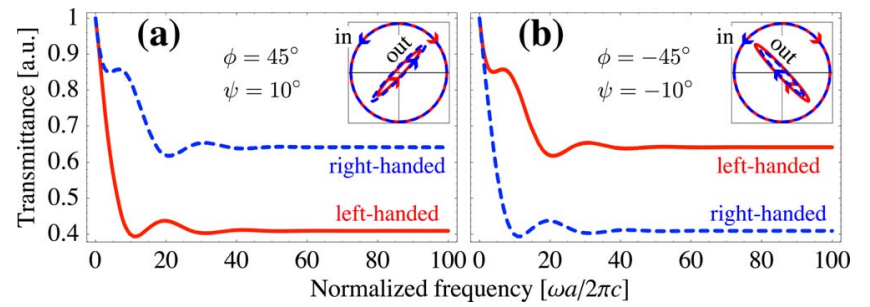

Fig. 4. (Color online) Transmission spectra for an LH-RH circularly polarized wave passing through a slab with thickness $a$ made of material as in Eq. (3) for $\phi= \pm 45^{\circ}, \psi$ $= \pm 10^{\circ}, \varepsilon_{o}=1.1, \varepsilon_{e}=1.0, \kappa=0.2$, along with the incident (in) and transmitted (out) wave polarization at $\omega a / 2 \pi c=100$.

If the material is replaced with its enantiomeric counterpart $(\phi \rightarrow-\phi, \psi \rightarrow-\psi)$, or if the wave propagates in the opposite direction $(\mathbf{q} \rightarrow-\mathbf{q})$, the LH and RH plots are exchanged (Fig. 4). This agrees with experimental results for PCSs [13] and with the Lorentz homogenization scheme.

When an initially circularly polarized wave travels through the layer, the wave is seen to assume the polarization matching one of the eigenstates in orientation and both eigenstates in handedness. So, a wave whose handedness does not match that of the eigenstates undergoes polarization conversion, as reported earlier [7]. This can be understood from the combined action of anisotropy (creating $\mathrm{LH} \leftrightarrow \mathrm{RH}$ polarization mixing) and dichroism (diminishing the circular polarization component not matching the eigenstates in handedness).

The results obtained are complementary to a recent account [13] linking planar chirality with polarization-sensitive excitation of resonant modes in CSRs. There, e.g., LH and $\mathrm{RH}$ waves were shown to excite an electric dipole (well coupled to the field) and a magnetic dipole (poorly coupled to the field), respectively. For the latter, the energy is trapped in the resonant mode and dissipates, resulting in circular dichroism. The range of PCS designs where dichroism is brought about by this mechanism remains to be determined. It is also interesting to note that in high- $T_{c}$ anyon superconductors circular dichroism was reported [19], yet no sign of Faraday-like nonreciprocity was found [20], similar to PCSs. This striking similarity may be attributed to the layered structure of such superconductors.

To summarize, we have shown that bulk media with elliptical dichroism exhibit optical properties characteristic for PCSs. First, the elliptical polarization eigenstates are corotating (Fig. 3). Second, transmission for a LH-RH-polarized incident wave is asymmetric and is exchanged if the material is replaced with its enantiomeric counterpart (Fig. 4). Finally, a wave propagating in such a material undergoes circular polarization conversion so as to match the eigenstates. The medium is described solely by a complex symmetric dielectric tensor whose structure is given by homogenization of a split-ring PCS. This way, it is shown that a crystallographic approach is possible in studying PCSs, offering a theoretical starting point for analyzing planar chirality as an optical phenomenon, e.g., in bianisotropic multilayer solvers [21].

The authors acknowledge helpful suggestions from V. Fedotov, E. Plum, and D. N. Chigrin. This work was supported in part by the Deutsche Fouschungsgemeinschaft (DFS) (FOR 557) and the Basic Research Foundation of Belarus (F08MS-006).

\section{References}

1. V. M. Shalaev, Nat. Photonics 1, 41 (2007). Science 322, 384 (2008).

2. M. Kuwata-Gonokami, N. Saito, Y. Ino, K. Jefimovs, T. Vallius, J. Turunen, and Y. Svirko, Phys. Rev. Lett. 95, 227401 (2005).

3. A. Papakostas, A. Potts, D. M. Bangall, S. L. Prosvirnin, H. J. Coles, and N. I. Zheludev, Phys. Rev. Lett. 90, 107404 (2003).

4. M. Decker, M. W. Klein, M. Wegener, and S. Linden, Opt. Lett. 32, 856 (2007).

5. E. Plum, V. A. Fedotov, A. S. Schwanecke, and N. I. Zheludev, Appl. Phys. Lett. 90, 223113 (2007).

6. C. Rockstuhl, C. Menzel, T. Paul, and F. Lederer, Phys. Rev. B 79, 035321 (2009).

7. V. A. Fedotov, P. L. Mladyonov, S. L. Prosvirnin, A. V. Rogacheva, Y. Chen, and N. I. Zheludev, Phys. Rev. Lett. 97, 167401 (2006).

8. V. A. Fedotov, A. S. Schwanecke, N. I. Zheludev, V. V. Khardikov, and S. L. Prosvirnin, Nano Lett. 7, 1996 (2007).

9. V. A. Fedotov, V. V. Khardikov, S. L. Prosvirnin, Y. Chen, and N. I. Zheludev, Nano Lett. 8, 2940 (2008).

10. E. Plum, J. Zhou, J. Dong, V. A. Fedotov, T. Koschny, C. M. Soukoulis, and N. I. Zheludev, Phys. Rev. B 79, 035407 (2009).

11. M. Reichelt, S. W. Koch, A. V. Krasavin, J. V. Moloney, A. S. Schwanecke, T. Stroucken, E. M. Wright, and N. I. Zheludev, Appl. Phys. B 84, 97 (2006).

12. A. Drezet, C. Genet, J.-Y. Laluet, and T. W. Ebbesen, Opt. Express 16, 12559 (2008).

13. E. Plum, V. A. Fedotov, and N. I. Zheludev, Appl. Phys. Lett. 94, 131901 (2009).

14. E. Plum, V. A. Fedotov, and N. I. Zheludev, Appl. Phys. Lett. 93, 191911 (2008).

15. E. Plum, X.-X. Liu, V. A. Fedotov, Y. Chen, D. P. Tsai, and N. I. Zheludev, Phys. Rev. Lett. 102, 113902 (2009).

16. J. Jackson, Classical Electrodynamics (Wiley, 1975).

17. F. I. Fedorov, Theory of Gyrotropy (Nauka, 1976), in Russian.

18. G. N. Borzdov, J. Math. Phys. 38, 6328 (1997).

19. K. B. Lyons, J. Kwo, J. F. Dillon, Jr., G. P. Espinosa, M. McGlashan-Powell, A. P. Ramirez, and L. F. Schneemeyer, Phys. Rev. Lett. 64, 2949 (1990).

20. S. Spielman, K. Fesler, C. B. Eom, T. H. Geballe, M. M. Fejer, and A. Kapitulnik, Phys. Rev. Lett. 65, 123 (1990).

21. S. V. Zhukovsky and V. M. Galynsky, J. Opt. A, Pure Appl. Opt. 8, 489 (2006). 